\title{
Endotoxin activity dosage in septic patients
}

\author{
Tito Del Gaudio', Maria Antonietta Distasi', Mariella Porzio', Adriana Mosca², Giuseppe Miragliotta² \\ I Laboratorio di Analisi P.O. Andria AUSL BAT \\ 2 Sez. di Microbiologia, Dip. MIDIM, Università degli Studi di Bari
}

Key words: Endotoxin, LPS, Toraymixin.

\section{Dosaggio dell'attività endotossinica in pazienti in stato settico}

\section{SUMMARY}

Endotoxin (LPS), present in the outer membrane of Gram negative bacteria, is responsible for a series of biological effects, primarily linked to the lipid fraction (Lipid A). The aim of our work was to correlate the endotoxin activity with the risk of severe sepsis in patients showing severe bacterial infection and hospitalized in the three Intensive Care Units of the AUSL BAT. Fifty-three endotoxin activity (EA) blood tests were performed on 28 septic patients. 17/28 (60.7\%) patients showed EA > 0.60 (associated with high risk of severe sepsis). Endotoxin activity (EA) blood tests were repeated in I I/I7 cases after LPS removal by extracorporeal blood treatment with Polymyxin B (Toraymyxin). 6/II patients had a reduction of LPS in the bloodstream after I treatment with Toraymyxin; in 5 of these 6 patients there was a further reduction after 2 treatments with Toraymyxin. EA dosage has been proven either a method for the diagnosis of severe sepsis due to Gram negative bacteria or helpful means in selecting patients for treatment with Toraymyxin.

\section{INTRODUZIONE}

Il Lipopolisaccaride (LPS) presente nella membrana esterna dei batteri Gram negativi produce nell'ospite, essenzialmente per la sua porzione lipidica (Lipide A), una serie di ripercussioni sul piano clinico che, sia pure con diversa intensità, si manifestano qualunque sia il batterio Gram negativo responsabile del processo infettivo. Il meccanismo alla base dell'azione tossica è il risultato della stimolazione di mediatori (di cui i principali sono TNF ed IL-1) che, attivando la via metabolica dell'acido arachidonico, stimolano la produzione di prostaglandine. Queste ultime, agendo sugli epiteli, causano aumento della permeabilità vascolare, vasodilatazione, ipotensione e shock emodinamico. Scopo del nostro lavoro è stato quello di correlare l'attività endotossinica dimostrabile, con il rischio di insorgenza di sepsi severa in pazienti affetti da infezione batterica grave e ricoverati nelle tre U.O. di Rianimazione dell'AUSL BAT. Ciò al fine di indirizzare ad una corretta selezione dei pazienti nei quali impostare e monitorare terapie basate sulla neutralizzazione delle endotossine.

\section{MATERIALI E METODI}

Nel periodo maggio 2008 - maggio 2009, 28 pazienti settici sono stati sottoposti a 53 dosaggi ematici di attività endotossinica (EA) mediante il test Endotoxin Activity Assay ESTOR (EAA®). L’LPS, eventualmente presente nel campione di sangue intero, reagisce con un anticorpo anti-LPS. L'immunocomplesso che ne deriva stimola i neutrofili a produrre radicali dell'ossigeno. Tale fenomeno è amplificato dalla presenza di Zymosan. I radicali prodotti reagendo con un luminoforo (Luminol) emettono luce, misurabile con un luminometro, di intensità proporzionale alla quantità di LPS presente nel campione. I valori espressi in Attività Endotossinica (EA) possono variare da 0 ad 1 . Il dosaggio dell'LPS dopo trattamento del sangue intero (Toraymyxin) è stato ripetuto per 11 di 17 pazienti che avevano riportato valori di EA > 0.60 (alto rischio di sepsi severa). Tale sistema consiste in una cartuccia per emoperfusione costituita da un involucro di policarbonato contenente tessuto di fibre di polistirene sulle quali è legata, mediante legame covalente, Polimixina-B. Durante il trattamento, il sangue intero perfonde la cartuccia e la Polimixina-B lega a sé e neutralizza l'endotossina presente.

\section{RISULTATI}

In 4/28 (14.3\%) pazienti è stato riscontrato $\mathrm{EA}<0.39$ (esclusione di sepsi da Gram negativi); in 7/28 (25.0\%) pazienti EA compreso tra 0.40 e 0.59 (rischio medio di sepsi severa) ed in 17/28 (60.7\%) pazienti EA > 0.60 (alto rischio di sepsi severa). In 6 degli 11 pazienti ad alto rischio di sepsi severa ed il cui sangue intero è stato sottoposto a trattamento (Toraymyxin), si è avuta una riduzione di LPS in circolo dopo 1 solo trattamento (Figura I); in 5 di questi 6 pazienti si è osservata una ulteriore riduzione dopo 2 trattamenti con Toraymyxin (Figura II).

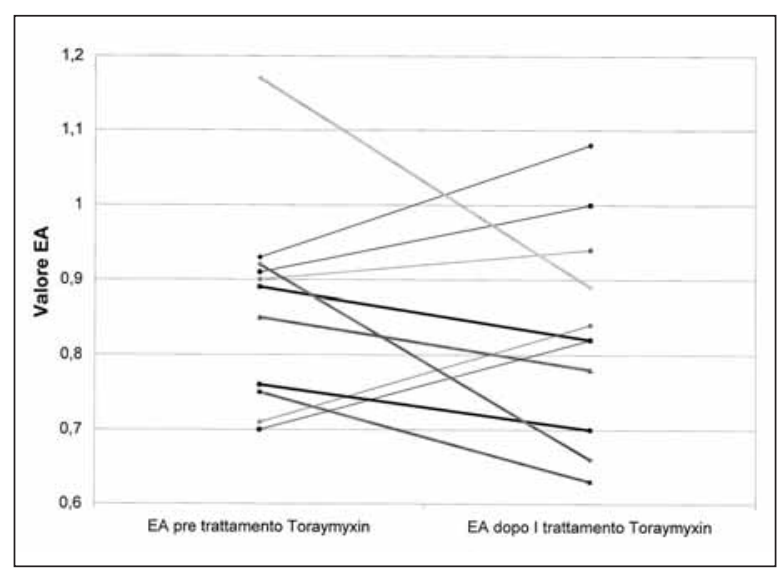

Figura I. Valori di EA (Attività endotossinica) dopo I trattamento con Toraymyxin.

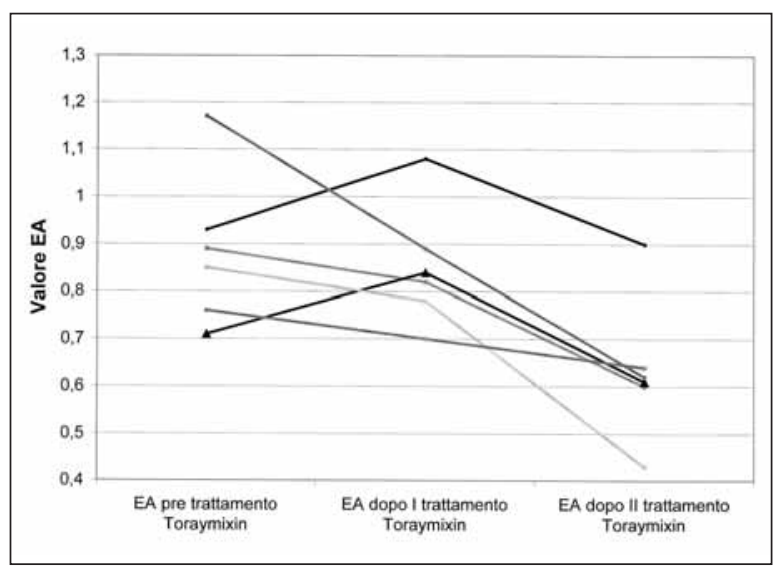

Figura II. Valori di EA (Attività endotossinica) dopo 2 trattamenti con Toraymyxin.

\section{Corresponding author: Tito Del Gaudio}

P.O. Andria AUSL BAT, Laboratorio Analisi

7003 I ANDRIA (BT) - Via R. Sanzio I6 - cell.: 3496376496 - Fax: 0883299 II 8

E-mail: titodelgaudio@tiscali.it 


\section{CONCLUSIONI}

Il dosaggio di LPS si è rivelato, nella nostra esperienza, un metodo utile per la diagnosi di sepsi severa da batteri Gram negativi. La più utile applicazione è inoltre quella di individuare tempestivamente i pazienti a rischio di sepsi severa da avviare al trattamento extracorporeo con Toraymyxin; nonché il loro monitoraggio dopo il trattamento stesso.

\section{BIBLIOGRAFIA}

1. Cruz DN, Cantaluppi V, Assenzio B, et al. Polymyxin-B hemoperfusion inactivates circulating proapoptotic factors. Intensive Care Medicine 2008; 34: $1638-45$.
2. Cruz DN, Perazella MA, Bellomo R, et al. Effectiveness of polymyxin B-immobilized fiber column in sepsis: a systematic review. Crit Car 2007; 11: R47.

3. Ronco C: The place of early haemoperfusion with polymyxin B-immobilized fibre column in the treatment of sepsis. Crit Care 2005; 9: 631-3.

4. Shigeatsu E, Nobuhiro S, Yasushi S, Masahiro K, Gaku T, Yasunori Y. Efficacy and Problem of a New Endotoxin Activity Assay Method. ICU and CCU. Japanese Journal of Intensive Care Medicine 2007: 31:407-10.

5. Shimada N, Nakamura T, Takayashi Y, et al. Effects of polymyxin Bimmobilized fiber on serum phosphate concentrations in patients with sepsis. Nephron 2000; 86: 359-60.

6. Shoji H. Extracorporeal endotoxin removal for the treatment of sepsis: endotoxin adsorption cartridge (Toraymyxin). Ther Apher Dial 2003; 7: 108-14.

7. Valenza F, Fagnani L, Coppola S, et al. Prevalence of endotoxemia after surgery and its association with ICU length of stay. Crit Care 2009;13: R102. 